Modeling of primary dendrite arm spacing variations in thin-slab casting of low carbon and low alloy steels

This content has been downloaded from IOPscience. Please scroll down to see the full text. 2012 IOP Conf. Ser.: Mater. Sci. Eng. 27012046

(http://iopscience.iop.org/1757-899X/27/1/012046)

View the table of contents for this issue, or go to the journal homepage for more

Download details:

IP Address: 134.83.1.242

This content was downloaded on 05/05/2015 at 15:13

Please note that terms and conditions apply. 


\title{
Modeling of primary dendrite arm spacing variations in thin-slab casting of low carbon and low alloy steels
}

\author{
H Mehrara ${ }^{1,2}$, B Santillana ${ }^{1,3}$, D G Eskin ${ }^{4}$, R Boom ${ }^{1,2}$, L Katgerman $^{1}$ and G Abbel ${ }^{3}$ \\ ${ }^{1}$ Department of Materials Science and Engineering, Delft University of Technology; Delft, \\ The Netherlands \\ ${ }^{2}$ Materials innovation institute M2i, Delft, The Netherlands \\ ${ }^{3}$ Tata Steel RD\&T, POBox 10000, 1970CA, IJmuiden, The Netherlands \\ ${ }^{4}$ BCAST, Brunel University, Uxbridge UB8 3PH, U.K.
}

Email: h.mehrara@tudelft.nl (mehrara@live.com)

\begin{abstract}
Solidification structure of a High Strength Low Alloy (HSLA) steel, in terms of dendrite arm spacing distribution across the shell thickness, is studied in a breakout shell from a thin-slab caster at Tata Steel in IJmuiden. Columnar dendrites were found to be the predominant morphology throughout the shell with size variations across the shell thickness. Primary Dendrite Arm Spacing (PDAS) increases by increasing the distance from meniscus or slab surface. Subsequently, a model is proposed to describe the variation of the PDAS with the shell thickness (the distance from slab surface) under solidifiction conditions experienced in the primary cooling zone of thin-slab casting. The proposed relationship related the PDAS to the shell thickness and, hence, can be used as a tool for predicting solidifcation structure and optimizing the thin-slab casting of low alloy steels.
\end{abstract}

\section{Introduction}

It is a general belief that quality of cast products is a direct consequence of a structure formed during solidification. Through extensive studies on solidification macro- and microstructure development, it is also well accepted that under most industrial casting conditions, the predominant structure comprises dendritic morphology of the solid phase. Although the development and evolution of the dendritic morphology are complex phenomena, the resultant dendritic microstructure has a characteristic feature called dendrite arm spacing, which has been long used as a measure of fineness of cast structure and, hence, quality of as-cast products.

Based on immense theoretical and experimental works, the spacing of dendrite arms has been linked to other solidification parameters like dendrite tip velocity, cooling rate, temperature gradient in the solidifying metal, local solidification time etc. [1] and, accordingly, many models have been proposed for both primary and secondary dendrite arm spacing; PDAS and SDAS respectively. In spite of geometrical constraints acting upon PDAS, its importance lies in suitability to reflect processstructure-properties correlations $[1,2]$. However, most studies on PDAS have been conducted under steady state unidirectional solidification conditions in terms of growth rate and temperature gradient, which are difficult to measure and control in industrial processes like continuous casting (CC).

The present study focuses on characterization of the dendritic structure in early stages of solidification of a steel shell inside a thin-slab continuous caster mold. The primary objective was to 
identify how primary dendrite arm spacing varies as the distance from the meniscus or the chill surface increases. To this end, solidification structure in a breakout shell of a grade of HSLA steels is first examined by measuring PDAS along the shell and through its thickness. A breakout shell is like a snapshot of the process, demarcating the solidified part from the liquid domain inside the mold. The interface between those two regions well represents the solidification front and development of dendritic structure within the mushy zone under casting conditions. Another objective was to derive a simple model, which can reflect functional dependency of PDAS on the distance from meniscus in the mold and from the surface of the solidified shell. The proposed model is based on available theoretical analysis of PDAS and is verified with the findings of breakout shell research. Finally, the model is also applied to completely solidified slabs of two other grades of steel.

\section{Experimental Procedure}

A breakout shell of Low-Range High Strength Low Alloy (LR-HSLA) steel, taken from a Direct Sheet Plant thin-slab continuous caster at Tata Steel in IJmuiden, was studied. Chemical composition of this steel is given in table 1 . History of processing parameters prior to shell outbreak such as casting speed, mold temperature etc. are known from operation records. Sampling scheme within an upper part of the shell is shown in figure 1. After surface preparation, specimens were etched using Bechet-Beauchard solution to reveal the cast structure. The microstructures were examined under the optical microscope.

Table 1. Chemical composition and casting speed of the steel grade in this study

\begin{tabular}{ccccccc}
\hline $\begin{array}{c}\text { Steel } \\
\text { grade }\end{array}$ & $\begin{array}{c}\mathrm{C} \\
(\mathrm{wt} \%)\end{array}$ & $\begin{array}{c}\mathrm{Mn} \\
(\mathrm{wt} \%)\end{array}$ & $\begin{array}{c}\mathrm{V} \\
(\mathrm{wt} \%)\end{array}$ & $\begin{array}{c}\mathrm{Nb} \\
(\mathrm{wt} \%)\end{array}$ & $\begin{array}{c}\mathrm{N} \\
(\mathrm{ppm})\end{array}$ & $\begin{array}{c}\text { Casting speed } \\
(\mathrm{m} / \mathrm{min})\end{array}$ \\
\hline LR-HSLA & 0.045 & 0.8 & 0.04 & 0.013 & 80 & 5.4
\end{tabular}

Measurement of PDAS was carried out along solidification front - at a given distance in order to offset microstructural changes due to drainage during breakout time - from the meniscus level down to the mold exit. Figure 1 illustrates a longitudinal section of the breakout shell along with measurement locations on the specimens shown by circles. At each location, an average of at least three measurements was taken as PDAS value there. The trajectory constructed by these circles indicates different solidification moments at the corresponding distances from the mold or slab surface where the alloy undergoes varying solidification conditions.

Correlation of structure formation with heat transfer evolution during solidification requires knowledge of shell buildup within the mold. The thickness of the breakout shell was measured with a 3D laser method developed for this purpose as reported in [3]. Moreover, the measurements of the PDAS for two other grades i.e. Low Carbon Aluminum Killed (LCAK) and High Strength Low Alloy (HSLA) steels were analyzed to verify the proposed relationship.

\section{Results}

An example of the solidification structure of the breakout shell studied here is shown in Figure 2. As seen in this micrograph, the predominant morphology is a columnar dendrite but with variable fineness across the specimen. Typically, the structure is noticeably finer near the solid surface in the vicinity of the chill mold (left edge of the micrograph) where cooling rate is higher. Such superficial region corresponds to the solidification in upper parts of the mold at closer distances to the meniscus while the solid forming at a larger distances from the surface experiences a lesser cooling rate corresponding to the lower part of the mold. 


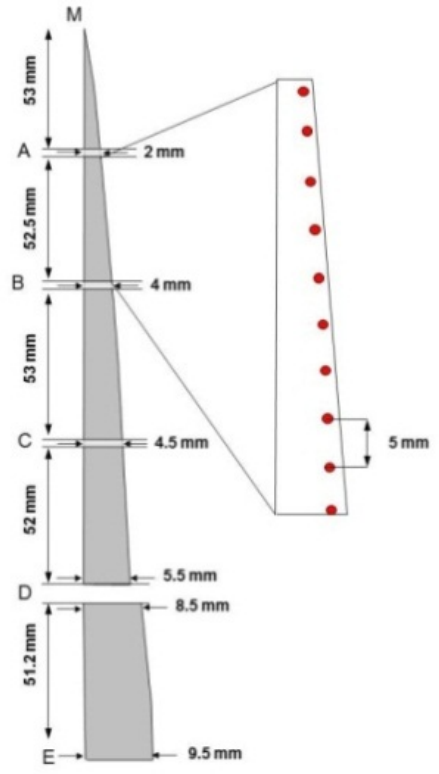

Figure 1. Sampling scheme in the examined piece of the breakout shell. The circles are measurement locations in the microstructure. ( $\mathrm{M}$ and $\mathrm{E}$ stand for meniscus level and mold exit direction, respectively, with the total distance $234.7 \mathrm{~mm}$ ).

Figure 3 depicts the result of the PDAS measurement and the shell thickness in the LR-HSLA breakout shell. As distance from the meniscus increases, both the shell thickness and the PDAS increase. In other words, build-up of the solid layer results in a coarser dendritic structure.

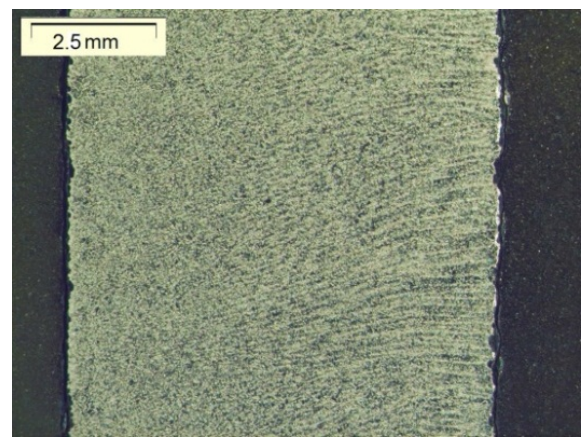

Figure 2. Optical micrograph of the solidification structure in of LR-HSLA breakout shell (surface is on the left).

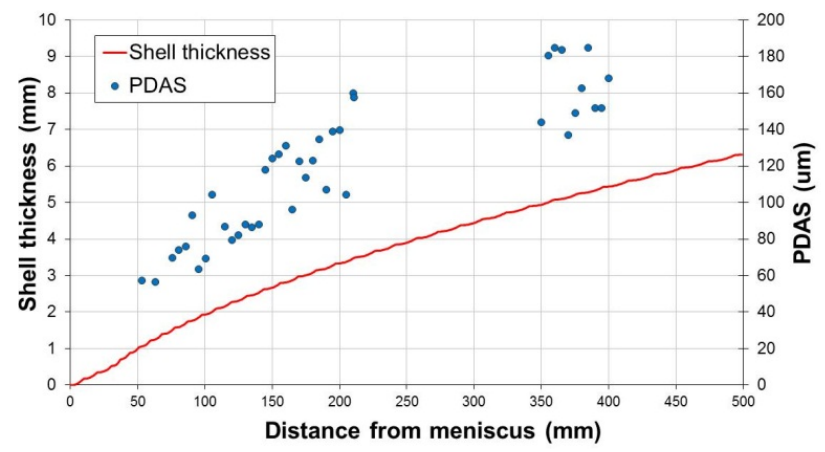

Figure 3. Shell thickness and PDAS as functions of the distance from the meniscus in the CC mold for the LRHSLA breakout shell.

\section{Model}

It is well established that microstructure developed in the solidification range depends on the thermal gradient $(\mathrm{G})$ and the growth rate $(\mathrm{R})$ of the solidification front [2]. The primary and secondary dendrite arm spacing $(\lambda)$ in terms of these variables are generally expressed in the following form:

$$
\lambda=\mathrm{CG}^{-m} \mathrm{R}^{-n},
$$

where $\mathrm{C}$ is a constant depending on alloy composition; and the exponents $m$ and $n$ indicate relative effect of a variation of $\mathrm{G}$ and $\mathrm{R}$ upon dendrite arm spacing. Theoretical models characterizing columnar growth adopted values of 0.5 and 0.25 for $\mathrm{m}$ and $\mathrm{n}$ respectively [4], while semi-empirical 
models suggest a range of values [5, 6]. For SDAS they are nearly identical whereas different for PDAS. Thus, PDAS will not obey a simple correlation - a unique function of cooling rate (G.R) or local solidification time - as usually found in the literature for SDAS.

In the present analysis, the functional relationship of the distance from the slab surface, as an easily measurable variable in continuous casting, to PDAS is sought. First, the general form of the primary dendrite arm spacing according to the theoretical model by Kurz and Fisher [4] postulates that $m=0.5$ and $n=0.25$

It is assumed that the shell thickness build-up is governed by a parabolic growth. The parabolic growth of solid shell is reported to be a good approximation for slab continuous casting [7]. It follows that

$$
\mathrm{x}=\alpha \sqrt{\mathrm{t}}=\beta \sqrt{\mathrm{z}},
$$

where $\mathrm{x}$ is shell thickness (distance from the slab surface), $\mathrm{t}$ is solidification time and $\mathrm{z}=\mathrm{Vt}$ (here $\mathrm{V}$ is the casting speed and $t$ is time) is distance from the meniscus in the mold, $\alpha$ and $\beta$ are parabolic shell growth constants which are functions of mold design [7]. With plotting the shell thickness versus square root of the distance from meniscus for the breakout shell studied here, as shown in Figure 4, the parabolic growth is a valid assumption within the mold of a thin-slab caster (from the meniscus to mold exit), except for an entrance length.

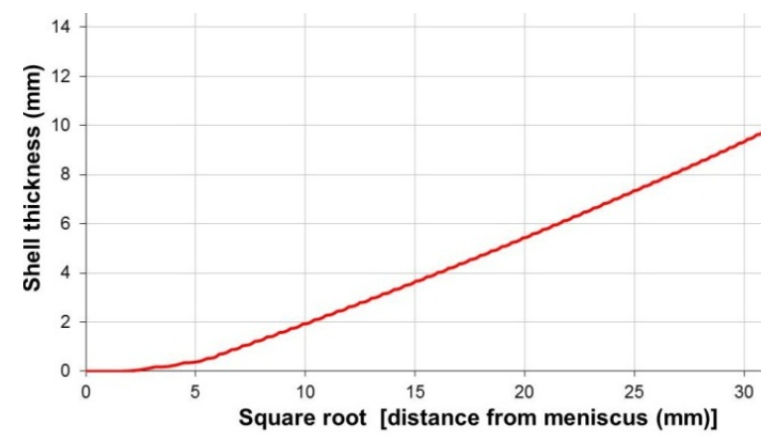

Figure 4. Solid thickness profile (of the breakout shell) as a function of distance from the meniscus

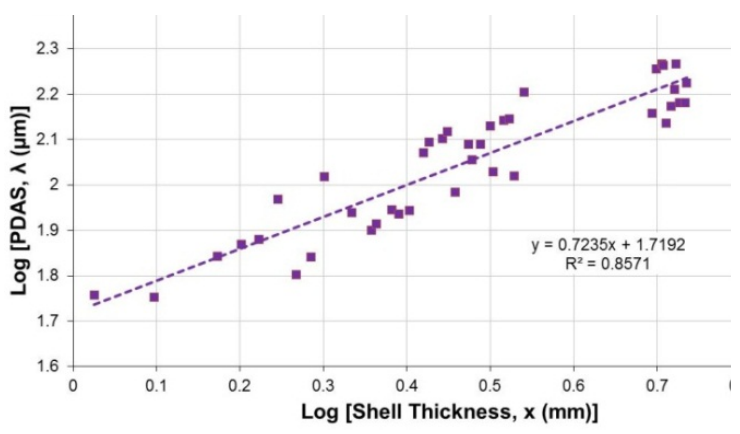

Figure 5. PDAS (in the breakout shell) as a function of distance from the solid surface (shell thickness).

Hanao et al. have investigated this entrance phenomenon in early stages of shell formation during continuous casting [8]. They pointed out that the parabolic growth is not exact due to a delayed period of solidification at the very beginning instances until the shell grows up to a given thickness. This delay and, in turn, the entrance region increase with increasing the casting speed. Kasama et al. showed that in this entrance region, the square root law has to be modified to take into account interfacial resistance effects [9]. These effects arise from dynamic changes at the metal-mold interface, for example, when the interface changes from a state of initial contact to a clearance gap [10].

After that particular position in the mold, the shell grows linearly with the square root of the solidification time (the so-called square root law, equation (2)). Thus, one can write taking into account equation (3) and making a simple transformation:

$$
\mathrm{R}=\frac{\mathrm{dx}}{\mathrm{dt}}=\frac{\mathrm{dx}}{\mathrm{dz}} \frac{\mathrm{dz}}{\mathrm{dt}}=\frac{\mathrm{dx}}{\mathrm{dz}} \mathrm{V}=\frac{\beta \mathrm{V}}{2 \sqrt{\mathrm{z}}},
$$

where $\mathrm{V}$ is the casting speed.

Under steady state solidification when temperature profiles are measured or calculated at a particular position during casting, both liquidus and solidus loci proceed in a similar parabolic fashion 
but each with its own constant i.e. $\beta_{\mathrm{L}}$ and $\beta_{\mathrm{S}}$ respectively. Thus, assuming a linear temperature gradient between liquidus and solidus and the thermal conductivity being a weak function of temperature:

$$
\mathrm{G}=\left.\frac{\mathrm{dT}}{\mathrm{dx}}\right|_{\text {mush }}=\frac{\mathrm{T}_{\mathrm{L}}-\mathrm{T}_{\mathrm{S}}}{\mathrm{x}_{\mathrm{L}}-\mathrm{x}_{\mathrm{S}}}=\frac{\Delta \mathrm{T}_{0}}{\sqrt{\mathrm{z}}}\left(\beta_{\mathrm{L}}-\beta_{\mathrm{S}}\right)^{-1},
$$

where $\mathrm{T}$ is temperature and subscripts $\mathrm{L}$ and $\mathrm{S}$ denote liquidus and solidus, respectively. Inserting expression equations (3) and (4) into equation (1), and recalling the parabolic growth of shell thickness along the mold wall (equation (2)), one can obtain

$$
\lambda_{1}=\left[\frac{1.19 \mathrm{C}}{\beta_{\mathrm{r}} \mathrm{V}^{\frac{1}{4}}}\left(\frac{\beta_{\mathrm{L}}-\beta_{\mathrm{S}}}{\Delta \mathrm{T}_{0}}\right)^{\frac{1}{2}}\right] \mathrm{X}^{\frac{3}{4}}=\mathrm{C}_{\lambda \mathrm{x}} \mathrm{x}^{\frac{3}{4}},
$$

where $\Delta \mathrm{T}_{0}$ is solidification range, $\beta_{\mathrm{L}}$ and $\beta_{\mathrm{S}}$ are shell growth factors for the liquidus and solidus loci respectively. $\beta_{\mathrm{r}}$ is representative shell thickness across the solidification range, which normally corresponds to coherency points at which a coherent dendritic network is developed. Therefore, the constant $\mathrm{C}_{\lambda x}$ encompasses material, processing and design parameters altogether. The calculation of $\mathrm{C}_{\lambda \mathrm{x}}$ requires thin-slab caster specifications - through careful measurement under steady state casting or by simulation using heat transfer/solidification codes - as well as knowledge of the solidification behavior of material within the freezing range; which is beyond the scope of this paper.

\section{Discussion}

Equation (5) expresses the functional relationship of the PDAS $\left(\lambda_{1}\right)$ to the distance from slab surface (x) for a solid growing along the $\mathrm{CC}$ mold wall provided that the square root law holds for growth. Mathematically, it follows that a graph of $\log \lambda_{1}$ versus $\log \mathrm{x}$ should be linear and have a slope of 0.75 . Figure 5 shows the PDAS as a function of the shell thickness (the distance from slab surface) for the LR-HSLA breakout shell of this study. The slope has a value of 0.72 very close to that predicted by the proposed model.

Using the $C$ value in equation (1) estimated using the data from [6] and the results of heat transfer simulation in thin-slab casting [3], PDAS can be calculated using the following relationship:

$$
\lambda_{1}=43.3 x^{\frac{3}{4}} .
$$

Note that the value 43.3 is close to the experimental value in figure $5(\log (43.3)=1.64$, close to 1.72$)$. Such model allows one to predict PDAS in thin-slab casting of this LR-HSLA steel by knowing the shell thickness and, hence, can be used as a tool to optimize design and processing parameters. Figure 6 compares the calculated (equation (6)) and measured values of PDAS at different distances from the breakout shell surface. It is to be noted that the application of this model to smaller thicknesses is restricted due to the violation of parabolic growth assumption. However, this approach could be extended to incorporate more complex heat transfer models for better prediction of growth rate during variable cooling conditions as experienced in real continuous casting processing and, hence, for a more accurate prediction of the PDAS values.

Santillana et al. [11] have measured the variations of PDAS with the distance from the surface for two fully solidified slabs of High Strength Low Alloy (HSLA) and Low Carbon Aluminum Killed (LCAK) steels. Re-construction of their findings in the form of log-log plot, as shown in Figure 7, reflects the same functional relationship, as proposed by the present model, for the primary zone of thin-slab casting. However, the specific correlation similar to equation (6) for these grades requires a complementary knowledge of alloy compositions, properties and casting conditions. 


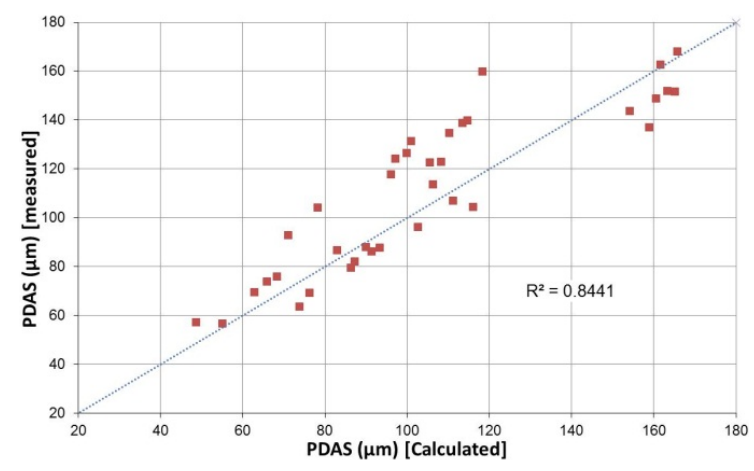

Figure 6. Comparison of the calculated versus the measured values for PDAS in the breakout shell

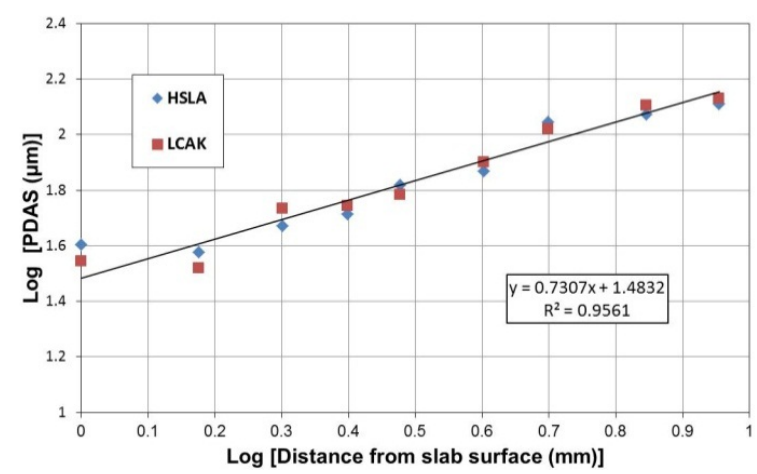

Figure 7. PDAS (in the solidified slabs) as a function of distance from slab surface

\section{Conclusions}

- Parabolic growth can well describe shell build-up in the mold region of thin-slab casting of low carbon-low alloy steel, except for solidification at the very beginning near the meniscus.

- The predominant structure of solidified shell inside the mold, as obtained by the breakout shell, is columnar dendritic.

- Primary arm spacing varies with the distance from the meniscus level (as in breakout shell) or distance from the solid surface (as in solidified slabs)

- The model proposes a functional relationship of PDAS to the shell thickness (the distance from slab surface) in the form of $\lambda_{1}=\mathrm{C}_{\lambda x} \mathrm{x}^{3 / 4}$ irrespective to the grade in the low alloy steels.

- Proportionality factor, $\mathrm{C}_{\lambda \mathrm{x}}$, depends on the steel composition, the solidification characteristics, the casting speed and the mold design.

\section{Acknowledgements}

This research was carried out under project number M41.5.08321 within the framework of the Research Program of the Materials innovation institute M2i (www.m2i.nl).

\section{References}

[1] Kurz W and Fisher D J 1998 Fundamentals of Solidification (Switzerland: TTP Pub.)

[2] Dantzig J A and Rappaz M 2009 Solidification (Laussane: CRC Press)

[3] Santillana B Thomas B G, Botman G, and Dekker E 2011 7th European Continuous-Casting Conference ECCC7 (Dusseldorf: MetTec InSteelCon) (CD)

[4] Kurz W and Fisher D J 1982 Acta Metallurgica 29 11-20

[5] Jacob J and Schwerdtfeger K 1976 Metallurgical Transactions A, 7A 811-820

[6] Taha H 1986 Journal of Materials Science Letters 5 307-310

[7] Shrewe H 1989 Continuous casting of steel (Germany: Stahleisen)

[8] Hanao M, Kawamoto M, and Yamanak A 2009 ISIJ International 49(3) 365-374

[9] Kasama A 1987 Near Net Shape Casting 59-63

[10] Ho K and Pehlke R 1985 Metallurgical and Materials Transactions B 16B(3) 585-594

[11] Santillana B, Eskin D G, Boom R, Katgerman L 2011 The 3rd International Conference on Advances in Solidification Processes ICASP3 (see current proceedings) 